\title{
Semiconductor Nanocrystals
}

\author{
Anurag Srivastava and Neha Tyagi
}

Additional information is available at the end of the chapter

http://dx.doi.org/10.5772/50440

\section{Introduction}

In terms of continuous miniaturization of electronic devices nanotechnology is a ray of hope for semiconductor industries due to peculiar properties of nano-materials that changes significantly the efficiency of the devices. Research in nanoscale materials get started because of the unique properties that are obtain at this scale, by changing the shape or size of these materials. At nanoscale, the behavior of materials drastically get changed and hence their properties. Particularly in semiconductors, it results due to the motion of electron to a length scale that is equal or smaller to the length scale of the electron Bohr radius that is generally a few nanometers. Continuous efforts are being made to explore the new physical properties of materials and to engineer them to fit for various technological applications. Scientific community has paid much attention to study various aspects of variety of nanostructured materials like; fullerenes, nanotubes (NT), nanoribbons (NR), nanowires (NW) and most recently, the nanocrystals (NCs). As the name indicates, NCs are the structures exhibiting crystalline structure but with one, two or all the three dimensions within the range of 1-100 nm. NCs enjoys advancement of crystalline periodicity at nano regime $\left(10^{-9} \mathrm{~m}\right)$ and often possesses new properties, most of the time reverse from those of the equivalent bulk materials due to their large surface to volume ratio, quantum size effect, and confinement effects [1-3]. Quantum confinement effect is the main phenomena that often been observed in NC, which deals with the spatial inclusion of the electronic charge carriers within the NC. The quantum size effect is analogous to the well known quantum mechanical problem i.e when a particle is place in a box, where the energy partition between the levels raises as the dimensions of the box reduces. Due to the same, we can observe an increment in the energy band gap of the semiconductor as the size of the crystal reduces. Nanostructuration is not confined to human imagination only; in fact it's an important choice of nature. The word nanotechnology is not new for the nature, where it has been realized for over billions of years which can be for instance in clays, circumstellar, dusts or many biological systems too. One of the most interesting examples of nature inspired nanotechnology is the water proof leaves of lotus owing to which a lotus can sustain in water for a very long time. Recently, it has been verified that lotus leaves has a special coating of 
nanoparticles which behaves as water repellant, this water repellant property has motivated number of researchers to perform research in this area and some of them even succeeded to manufacture hydrophobic coatings [4]. Interaction at the nanoscale are usually governed by the fact that the characteristic nano-length becomes comparable with other critical lengths of the system as mean free paths, scattering or coherence lengths. At the same time, confinement translates into a reorganization of the electronic density of states towards more distinct states. These two consequences of nanostructuration and their combination lead to the so called quantum related effects which determines most of the peculiar properties of nano-materials. A major corollary of nano confinement is the elevated ratio of the number of surface to volume atoms, which becomes another key point for the understanding of these extraordinary properties. In small NCs the ratio of the number of surface to the total number of atoms is less than one, for which it scales as $\sim 1 /(\text { Particle diameter })^{3}$ and exactly one in nanotubes or fullerenes, whereas in bulk crystal it goes to $10^{-20}$ or less.

NCs are very tiny crystals that results when a single crystal experience a solid to solid phase transition. Generally, NCs can be prepared by a huge number of atoms presented on the surface and no interior defects, unlike bulk materials. We all know that under the application of pressure abrupt change in the arrangement of the atoms i.e. structural transformation from one phase to another has been observed in crystals. The understanding of pressure-induced phase transition in various nanomaterials plays an important role in probing the properties of new materials. The consequences of finite crystal size on the structural phase transition and bulk modulus as well as compressibility has widely been demonstrated to get better understanding towards the stability, electronic, mechanical as well as other properties of nano-materials. Difference in surface energies of the two crystal phases is mainly responsible for the change in transition pressure in case of nano-materials. In fact the general rule states that smaller the size of the crystal, higher the transition pressure has been verified by several systems [5,6]. A report shows that NC shape can be easily observed using transmission electron microscopy at atmospheric pressure [7].

\section{Applications}

The enormous potential of exploring the new sciences and technology at nanoscale, may impact on industrial productivity, realized all over the world to develop the new materials for variety of newer applications. NCs possess variety of applications in the field of electronics, opto-electronics and photonics, and also several biological (medical) treatments following are the few important applications of semiconductor NCs.

\subsection{Nanocrystals LEDs}

In the last two decades very basic and more efficient white light-emitting diodes (LEDs) have been formed by replacing phosphors (a colour converter) with CdSe based NCs that are integrated directly into the p-n junction [8]. CdSe NCs/nanorods emit linearly polarized light along the crystallites axis and the degree of polarization depends upon the surface to volume ratio of the NCs [9]. A hydride structure comprises of single layer of CdSe NCs accumulate at 
the top of an InGaN/GaN quantum well (QW) has been utilized as LED which provides $~ 10 \%$ color conversion efficiency. An inverted LED design is shown in Fig. 1(a), where an InGaN QW grow on the top of a thick p-doped GaN barrier and complete the structure with a thin n-type GaN cap layer (normally a QW is grown on top of the n-type layer). Electrical contacts have been deposited by electron beam evaporation using an inter-digitated mask that comprised of several different device-mesa and contact geometries, as shown in Fig.1(b) [10].
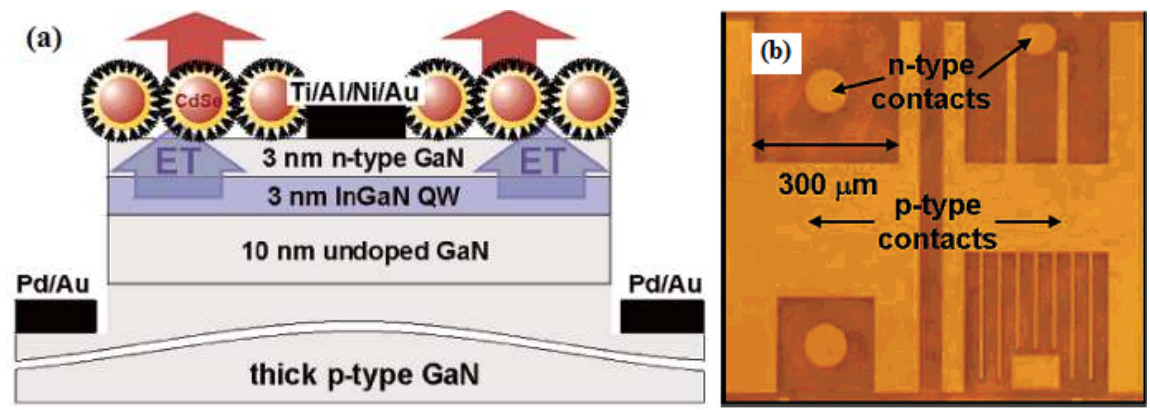

Figure 1. (a) Schematic of the nonradiative energy transfer (ET) LED structure. (b) Contact geometry of real device Ref.[10]

The conjugated polymers and InAs based NCs have been utilized to produce near infrared LEDs, where the emission can be tuned from the range of 1 to $1.3 \mu \mathrm{m}$ that efficiently covers the short-wavelength telecommunications [11]. Semiconductor NCs when excited electrically using $\mathrm{GaN}$ injection layer can be used as color selectable chromophores, shows high emission efficiencies with excellent photo stability and chemical flexibility. In a report by Mueller et al. [12] multicolour LEDs have been demonstrated where semiconductors NCs are integrated into a p-n junction made by GaN injection layers. Si NCs LEDs have been fabricated using ion implantation and observed the enhancement in LEDs optical output power ( 20 nW), with typical power efficiencies ranging from 1 to $10 \%$ [13]. Light emitting transistors integrated with individual CdSe NCs shows occurrence of Coulomb blockade at low bias voltage and low temperature, which signifies that electrons pass through the NC by single electron tunneling. Fig.2(a), shows a single nanorod contacted by two Au electrodes with about $30 \mathrm{~nm}$ separation. The representative current-voltage and electroluminescence (EL) data collected from a device "D1" is illustrated in Fig.2(b). A nonlinear increase in current $(I)$ at high bias voltage $(V)$ clearly reveals that the device exhibits a low-bias conductance gap. Once $V$ reaches a threshold $\left(V_{\text {th }}\right)$ the device starts emitting light as shown by EL intensity measurement [14].

\subsection{Nanocrystal memories}

Floating gate structure is firstly invented by Sze and Kahnd in 1967, which was used to construct flash memories [15] (Fig.3a). In 1995, IBM proposed first discrete NC memory and in early 2000's scientists have considered NCs as the capable candidate that can solve the current scaling problem (Fig.3b). The metal-oxide-semiconductor (MOS) memory structures based on Si NCs have potential applications in flash memory, reason being Si NCs are implanted as 
charge storage nodes in an oxide layer between the control gate and tunneling layer that reduces the difficulty of charge loss which can generally seen in conventional flash memories. Si NC provides faster write/remove speeds, small injection oxides, little operating voltages and better stamina [16]. Literature confirms that the information is stored in the NCs by injecting charges, due to which a transistor needs much voltage for turning it $\mathrm{ON}$, known as program operation illustrated in Fig.3(c). If we apply read voltage ( $\mathrm{V}_{\text {read }}$ ) to the gate between the program and erase operations to read the corresponding drain current $\left(\mathrm{I}_{\mathrm{d}}\right)$ then we obtain the memory status given by values 0 and 1 [17]. Generally the quantum dot/NCs flash memories have used Si NCs as a substitute to floating gate layer, however many results demonstrates the superiority of Ge based NC memories over those based on Si, because Ge NCs provides large nonvolatile charge conservation time because of their small band gap.
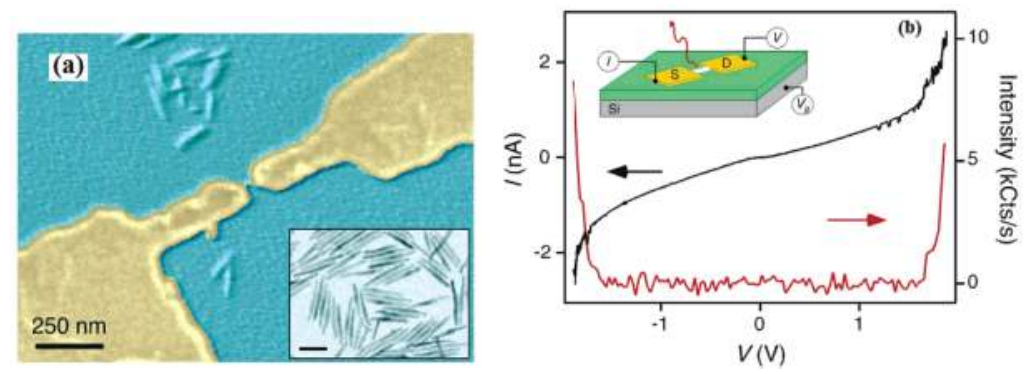

Figure 2. (a) SEM image (false color) of CdSe nanorod transistor. The inset shows a TEM image of the CdSe nanorods (scale bar is 50nm) (b) Current (black) and concurrently calculated EL intensity (red) plotted against bias voltage obtained from device. Inset illustrates the transistor model, with source (S) and drain (D) electrodes connecting the nanorod with a back gate is shown [Ref.14].

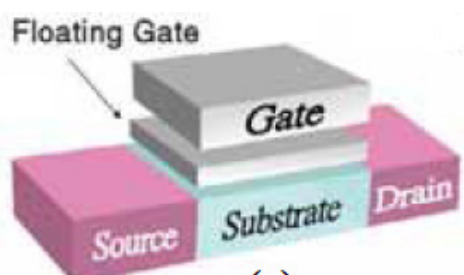

(a)

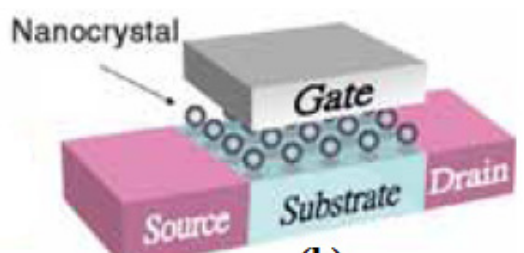

(b)

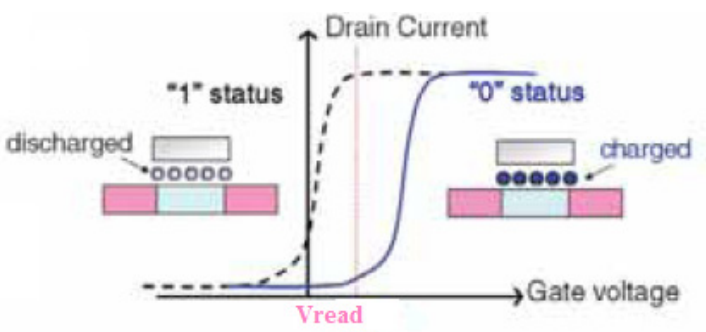

(c)

Figure 3. (a) Floating gate non volatile memory structure. (b) NC non volatile memory structure. (c) Program and erase mode of the NC memory device Ref.[17]. 
NCs may work as memory cells, where the conventional poly-silicon floating gate is replaced by an array of Si NCs, as the single cell and cell array of $1 \mathrm{Mb}$ and $10 \mathrm{k}$ have been realized by using a conventional $0.15 \mu \mathrm{m}$ FLASH technology [18,19]. Ge NCs have been fabricated by pulsed laser deposition for their use in floating gate memory, such memories shows excellent charge preservation characteristic. [20].

\subsection{Nanocrystal photocharge generators}

The PbSe NCs represent a fascinating system due to the easiness of knowing the quantum modulated optical nature in the infrared range with a Bohr exciton radius of $\mathrm{PbSe}$ around 46 $\mathrm{nm}$, where the quantum confinement effect come into view at comparatively large particle size. As we know that PbSe bulk crystal has rocksalt type phase and a direct band gap semiconductor $\left(\mathrm{Eg}_{\mathrm{g}}=0.28 \mathrm{eV}\right)$ whereas the PbSe NC show well defined band-edge excitonic transition, tuned from 0.9 to $2.0 \mathrm{eV}$ [21], leads to an efficient photocharge generators for communicating the IR wavelengths [22]. Highly efficient photo-detectors based on composites of the semiconducting polymer and PbSe NCs have been prepared and the outer quantum efficiency in these devices is larger than one [23]. Ge NCs photo-detector possesses powerful optical absorption and its photocurrent response has been measured within the wavelength range of 1.3 to $1.55 \mu \mathrm{m}$ with a low dark current of $61.4 \mathrm{nA}$ along with a a photocurrent responsivity of $56 \mathrm{~mA} / \mathrm{W}$ at the $5 \mathrm{~V}$ reverse bias [24].

\subsection{Nanocrystal solar cells}

An InAs/GaAs quantum dot solar cell has been synthesized and observed superior photocurrent without reduction of open circuit voltage compared to a solar cell without quantum dots. These solar cells have a light absorption range, which is extended up to 1.3 $\mu \mathrm{m}$ and confirms a trade-off between open circuit voltage and quantum dot ground state energy [25]. An ultrathin solar cell composed of CdSe and CdTe NCs of size 40nm shows 3\% power conversion efficiency and the device remain stable in air [26] (Fig.4).
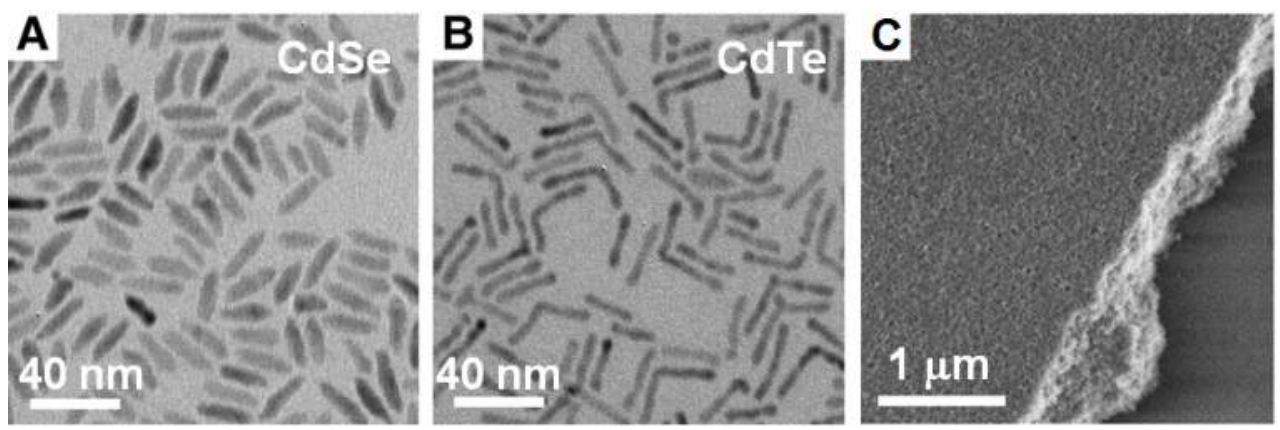

Figure 4. Transmission electron micrographs of (A) CdSe and (B) CdTe NCs (C) Spin-cast film of colloidal NCs imaged by scanning electron micrography is uniform and without defect; the film edge of this $\sim 100 \mathrm{~nm}$ film is shown for difference with the Si substrate Ref[26]. 
An induce electric field is large enough when two different molecules attached to a single $\mathrm{NC}$ and the field significantly changes the electronic as well as optoelectronic properties of the NC. By the help of mixed ligand, induced electric field significantly increases the efficiency of charge generation in CdSe NC solar cells, which enhanced the complete cell efficiency [27]. Si NC have quantum confinement property and so it can be used as photovoltaic materials since its ability to collect the photo-generated current through efficient electronic transport and for the development of Si NC based solar cells the exciton dissociation is a recent challenge [28].

\subsection{Nanocrystals in health}

Enzymes, membranes, nucleic acids, etc. are the elementary functional units made up of complex nanoscale particles in biological systems. In the era days of miniaturization where metals, semiconductors and magnets through which we construct optical and electrical sensors, can now be prepared on the scale of individual biological macromolecules that will have large impact on forthcoming medical treatments. In drug delivery and clinical applications, nanotechnology is one of the key factors for modern drug therapy. Due to the simplicity in preparation and general utility NCs are the new carrier-free colloidal drugs delivery systems having a particle size of $100-1000 \mathrm{~nm}$, these NCs have been thought of as a practical drug delivery approach to develop the poorly soluble drugs. Literature shows that the number of drugs coming directly from synthesis is nowadays poorly soluble and the drugs which are very less soluble in water combine very poorly with bioavailability. If we do not get any way to improve drug solubility then it will be very difficult to get absorbed from the gastrointestinal zone in the bloodstream and then to reach at the proper site for required action $[29,30]$. Recently intense research is being made on the colloidal quantum dots due to their stable light emitting nature, which can be broadly tuned by varying the size of the NC. Lots of efforts have been devoted in last two years on the development of large range of methods for bio conjugating colloidal NCs [31] because of their diverse applications such as in vivo imaging [32], cell tracking [33], DNA detection [34] etc. While studying the optical properties of NCs, it has been noticed that NCs fluorescence wavelength sturdily depends on their size as a result of which NCs photo bleach property get reduced. These NCs are fascinating fluorescence probes for various types of labelling research like cellular structures labeling, tracking the path and absorption of NCs by living cells [35].

\section{Synthesis \& characterization}

In general the performance of the material depend on its properties whereas the properties depend on the crystal geometry, constituents, defects and interfaces that are definitely prohibited by thermodynamics and kinetics of the fabrication process. In 1980's when the first theoretical explanation was proposed for colloidal spherical NCs by Brus [36], together with advances in the synthetic procedures [37] lead to a rapid increase in research in the field of nano sized materials. Following are the popular methods developed for the fabrication and characterizations of NCs: 
Solution-phase methods are being used for preparing CdS NCs with a wide variety of morphologies, starting with oleylamine as capping agent and by varying the reaction conditions [38]. Low temperature inverse micelle solvo-thermal route method has been used to synthesis large amount of single crystalline Ge NCs and X-ray diffraction measurement illustrates that these NCs are composed of pure cubic Ge structure. The size and morphology of the NCs are observed by transmission electron microscopy (TEM). In Fig.5(a) a TEM image of Ge NCs is illustrated and Fig.5(b) shows selected area electron area diffraction (SAED) sample for the NC of $25 \mathrm{~nm}$ size and displays the typical spot pattern of a single crystal domain and also the lattice planes of Ge with [111] hexagonal symmetry. High resolution TEM (HRTEM) images of high quality Ge NCs are shown in Fig. 5(c) and (d) [39].
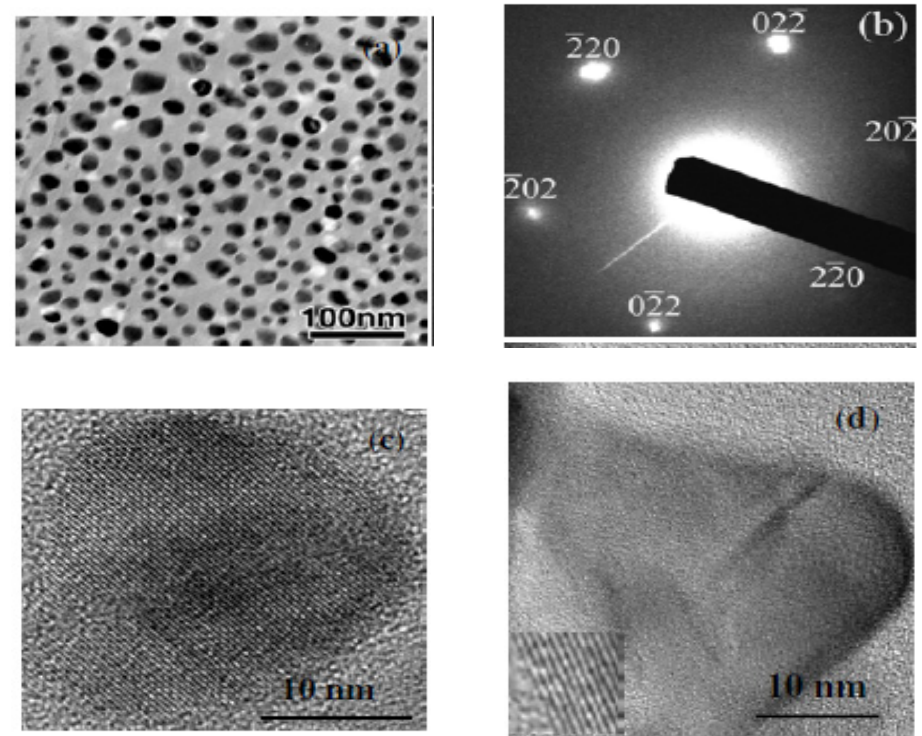

Figure 5. (a) As-prepared Ge NCs TEM image, (b) SAED sample of Ge NC with 25nm size; HRTEM images of spherical (c) and triangular(d) Ge NCs Ref.[39]

Few non-hydrolytic methods have also been developed for the synthesis of ZnO NCs with controlled size, shape and surface-defect at low temperature. But such methods need very cautious control of conditions and carefully engineered precursor.

Direct liquid phase precipitation is a new process proposed recently for NC fabrications, however slight hard from the previous complex fabrication methods in superficial and efficient way (at room temperature). This process produced perfect $\mathrm{ZnO}$ NCs of different diameter (5 to $12 \mathrm{~nm}$ ) and of various shapes [40].

Benzene thermal method at low temperature and low pressure $(0.12-0.14 \mathrm{MPa})$ has been used for the synthesis of AlN NCs. Owing to the catalytic effect of AlN nanocrystals, $8 \%$ benzene was converted at $150^{\circ} \mathrm{C}$ into several polymers, like cyclohexylbenzene [41]. 
Sol-gel technique can be used for the fabrication of amorphous or crystalline materials from a liquid phase at low temperature. This technique employed for the production of laser dyes, enzymes, nano sized semiconductors and metal nanoparticles. AlP NCs embedded in silica glasses have been prepared by sol gel process, where the preparation of gels take place by the complex solution hydrolysis and after about 10hours heating in an air atmosphere to form gel glass, again get heated in the presence of $\mathrm{H}_{2} \mathrm{~N}_{2}$ gas and react with $\mathrm{Al}(\mathrm{III})$, finally a cubic AlP crystallites are produced [42]. Similar technique has been used in producing InAs and $\mathrm{InP}$ microcrystallites embedded in $\mathrm{SiO}_{2}$ gel glasses [43,44]. GaAs NCs have been synthesized by an electrochemical route method from the acidic solutions of metallic gallium and arsenic oxide [45]. The growth of 1D nanostructures have traditionally been associated with such highly technological processes/analysis tools as vapor deposition, lithography, thermally assisted electromigration method [46], annealing process [47], scanning tunneling microscope (STM), atomic force microscope (AFM) [48]. By using size selective precipitation techniques, cadmium chalcogenide NCs have been successfully obtained [49]. The most common semiconductor materials grown by precipitation technique are CdTe, CdSe, CdS, and ZnS of which CdTe has special technological importance because it is the only known II-VI material that can form conventional p-n junctions [50]. For the synthesis of III-V compound semiconductors on the semiconductor substrate variety of methods have been developed starting from metaorganic chemical vapour phase epitaxy (MOVPE), molecular beam epitaxy (MBE) [51], various inorganic techniques [52], porous glass [53] to photo chemical vapour deposition [54].

In chemical vapour deposition (CVD), the vaporized precursors are introduced into a CVD reactor and adsorbed onto a substance held at high temperature. These adsorbed molecules will either thermally crumble or react with other gases to form crystals. The CVD process has been organised in three steps (i) mass transport of reactants to the growth surface through a boundary layer by diffusion (ii) chemical reactions on the growth surface, and (iii) removal of the gas-phase reaction by products from the growth surface. NCs have been characterized by X-ray diffraction, scanning transmission electron microscopy (STEM), optical absorption spectroscopy and fluorescence spectroscopy.

Keeping in view of all the techniques discussed for the synthesis and characterization of NCs we can state that in current scenario it has been easier task to fabricate a NC of desired morphology.

\section{Properties}

\subsection{Structural properties}

The structural properties of nanocrystals have been performed using various experimental as well as theoretical approaches. In general, a structural property means the analysis of stability of the crystal structure and then the evaluation of ground state parameters such as bond length, bond angle, lattice parameter etc. For example a periodic cluster approach with an atomistic pair potential has been employed to simulate AlN NC and predictes that the NCs displays graphitic-like layers and non-buckled wurtzite structure. The variation of surface 
tension, c/a ratio, and equation of state for AlN NC with respect to both the pressure and size has also been examined. The study also reveals that the bulk modulus of the wurtzite phase of NC is smaller (although decreasing with size) than its bulk counterpart [55]. Recently, Franceschetti [56] has analyzed the structural and electronic properties of PbSe NCs with different radius. Fig.6 shows the optimized structures of PbSe NCs in Pb44Se $\mathrm{Se}_{44}, \mathrm{~Pb}_{140} \mathrm{Se}_{140}$ and $\mathrm{Pb}_{240} \mathrm{Se}_{240}$ form and both $\mathrm{Pb}$ and Se atoms have rocksalt structure. The Pb-Se bond lengths and bond angles are appreciably distorted contrast to that of its bulk counterpart as represented in Fig.7. In fact, Fig.7 clearly shows the deviation of bond length of the Pb-Se with respect to the distance of the bonds from the center of the NC and the bond lengths of Pb-Se NCs are in close match with its bulk crystal bond length (3.103 $\AA$ ). The average Pb-Se bond length of the NC in the ground state is smaller than the bulk crystal bond length and decreases with the size. The formula for calculating formation energy of $\mathrm{NC}(\mathrm{PbSe})$ can be given as

$$
\Delta \mathrm{E}_{\mathrm{F}}=\mathrm{E}_{\mathrm{NC}}-\mathrm{NE}_{\text {bulk }}
$$

where the calculated total energy for NC is ENC with calculated bulk crystal total energy Ebulk and total number of $\mathrm{Pb}$ and Se atoms are represented by $\mathrm{N}$ for $\mathrm{NC}$.
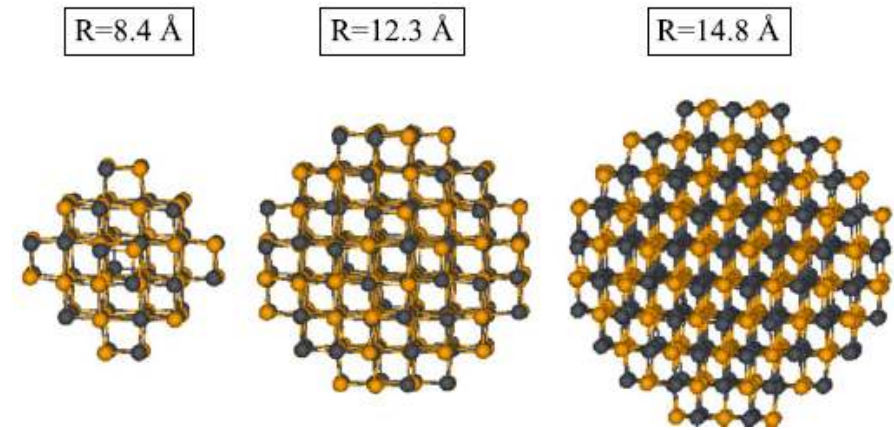

Figure 6. Three different sizes optimized geometries of PbSe NCs. The dark circles indicate $\mathrm{Pb}$ atoms and the light circles for Se atoms [56].

Srivastava et al. have also investigated the structural properties of various bulk as well as NCs such as AlAs [57,58], AlSb [59] and AlN [60]. The structural properties of bulk AlAs in zincblende (B3), wurtzite (B4), NiAs (B8), $\mathrm{CsCl}$ (B2) and $\mathrm{NaCl}$ (B1) type phases have been analyzed through first principle density functional theory (DFT) approach. Using local density approximation ( LDA) with Perdew-Zunger (PZ) type parameterization the equilibrium lattice constant (a) of original B3 type phase of bulk AlAs is calculated as $5.64 \AA$ and also from GGA-PBE (5.72 $\AA$ ) and GGArevPBE (5.77 $\AA$ ). The stability analysis of bulk AlAs in $\mathrm{B} 8$ type phase, corresponds to the $c / a$ ratio of $1.59 \AA$, where the equilibrium lattice constants $a$ and $c$ are $3.72 \AA$ and $5.91 \AA$, respectively [57]. Similar approach has been used for analyzing the structural stability of AlAs NCs in B3, B4, B2 and B1 type phases at $\sim 0.9 \mathrm{~nm}$, where the lattice parameters for all the four stable phases of AlAs NCs [58] such as B3, B4, B1 and B2 are 5.761, 4.283, 5.350 and $3.207 \AA$, respectively. The total energy values for the B3, B4, B1 and B2 type phases of AlAs NCs are $-468.52,-468.46,-467.84$ and $-467.77 \mathrm{eV}$, 
respectively. Fig. 8 shows the variation of energy with respect to volume for B3 and B1 type phase of AlAs NCs. The calculated lowest total energy confirms that the B3 phase as the most stable one at lower pressure and the B1 type phase can be considered as the high pressure phase. On comparison with bulk AlAs, stability trend of the low pressure phases are same in bulk and nano-dimension but the high pressure phases are different.

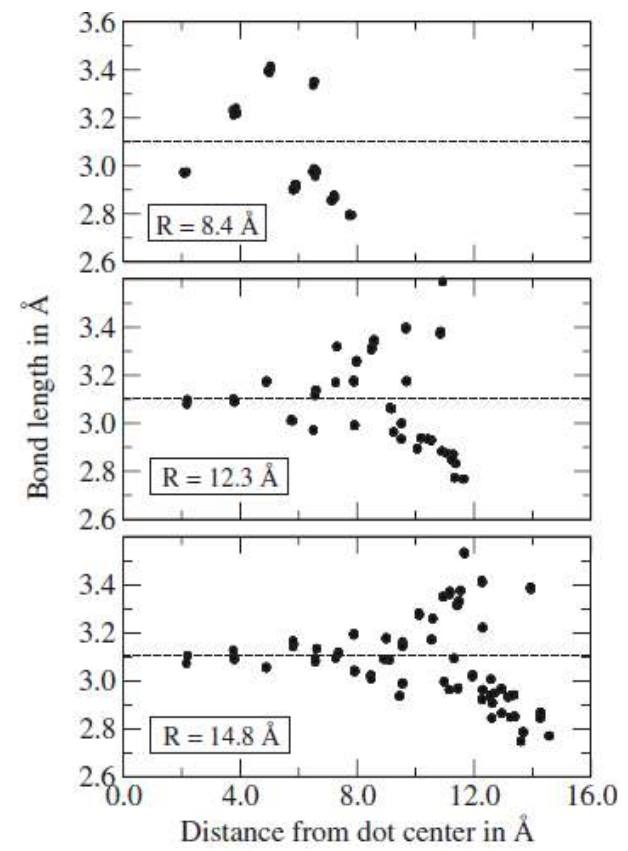

Figure 7. The variation of all $\mathrm{Pb}$-Se bonds as a function of distance of the bond from the geometric centre of the NC. The dash lines shows the computed bulk Pb-Se bond length Ref.[56].

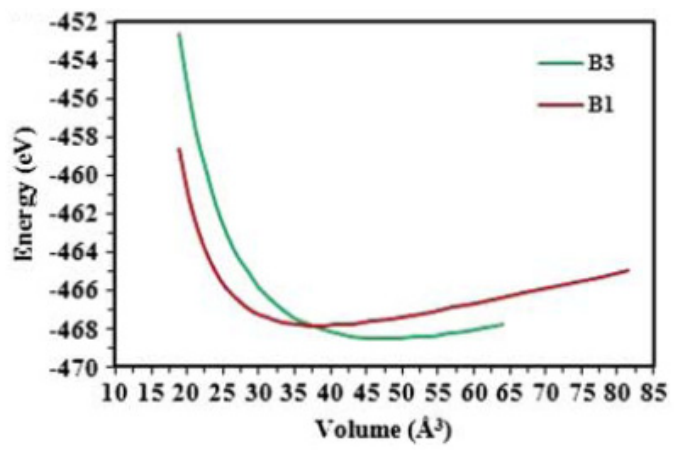

Figure 8. Energy as a function of volume for AlAs nanocrystals Ref.[58].

The stability of AlSb nanocrystal of $~ 1 \mathrm{~nm}$ size has also been analyzed in B3, B1 and B2 type phases theoretically. The article reports that computed lattice constant (6.235 ̊) for bulk 
$\mathrm{AlSb}$ in B3 type phase is in close match with its experimental as well as other theoretical counterparts. However the computed lattice constants of B3, B1 and B2 type phase of AlSb NCs are $5.33 \AA, 5.92 \AA$ and $3.57 \AA$ respectively. The findings conclude that B3 type phase is most stable with lowest total energy and highest binding energy [59]. In an another report by Tyagi et al. ab-initio pseudopotential approach has been used for calculating the lattice constants of 1-D AlN NC in B3, B1 and B2 type phase as $4.58 \AA, 4.48 \AA$ and $2.98 \AA$ respectively [60]. Besides these theoretical works few experimental work has also been performed as zinc-blende GaP NCs have been synthesized by three different methods and it has been found that NCs transferred irreversibly into four new phases of GaP NCs with different lattice constants $(5.8872,5.5493$ and $7.0339 \AA$ ) and the largest one is about 1.3 times of the reported data, which has been characterized by X-ray diffraction and transmission electron microscopy [61].

\subsection{Electronic properties}

Electronic structures in nano regimes are extremely enviable for the future potential electronic devices. On the other hand, nano structures are still a challenge, where different experimental as well as theoretical approaches have been employed to understand the variation of electronic structures of NCs as a function of its size. The study of quantum confinement phenomena in semiconductor NCs is the subject of intense research which in fact determines their electronic behavior. A theoretical approach effective mass approximation (EMA) [62] is employed firstly to understand quantum confinement effects on the electronic band gap as a function of size of NCs. The confining potential for the electron and hole are assumed infinite in most of the EMA calculations. In EMA approach, the wave function of both electron as well as hole get vanishes at and beyond the surface of the NCs without any tunneling. To date numerous theoretical methods have been developed such as first-principles [63], semiempirical pseudopotential [64] and tight binding (TB) method [65] to see the effect of size on the electronic band structure of various NCs. The TB method has received great attention because of its realistic description of structural and dielectric properties in terms of chemical bonds and ease of handling large systems. For the description of hole wave function the effective bond orbital model and EMA calculations are used for of electron wave function by Einevoll [66], and Nair and his coworkers [67]. To see the effect of NC size on the electronic band structures various pseudopotential and TB calculations have been performed. UV-visible absorption spectroscopy tool has been utilized to study the variation of band gap as a function of NC size. There are reports on the individual shifting of highest occupied molecular orbital of the valence band and the lowest unoccupied molecular orbital of the conduction band region with NC size, using various methods of energy spectroscopy e.g. X-ray absorption and photoemission [68]. Another semi empirical pseudopotential method has been employed to calculate the electronic structures of $\mathrm{Si}, \mathrm{CdSe}$ [69] and InP [70] nanocrystals. An appreciable progress has been observed in the accuracy and the results of the electronic band structure calculations of NCs while using TB and pseudopotential approaches. In comparison to EMA, the reason behind the popularity of TB might be low computational effort, basic picture of atomic orbitals and 
hopping interactions for a predefined range. The ab-initio electronic band structure computation may lead to perceptive TB scheme and show accuracy because it use physical and real basis set. In 1989 Lippens and Lannoo [71] have used semi-empirical TB approach with $\mathrm{sp}^{3} \mathrm{~s}^{*}$ orbital basis to compute the variation in the energy gap of semiconductor NCs as a function of size and the results so obtained are in good agreement with the EMA potential, however the $\mathrm{sp}^{3} \mathrm{~s}^{*} \mathrm{~TB}$ model have a tendency to underestimate the energy gap. This model cannot reproduced the lowest lying conduction band, however the electron-hole interaction improves the nearest neighbor $\mathrm{sp}^{3} \mathrm{~s}^{*}$ model by the inclusion of spin-orbit coupling. In a study on InP [72] NCs where the $\mathrm{sp}^{3} \mathrm{~d}^{5}$ orbital basis is used for the anion and the $\mathrm{sp}^{3}$ basis for the cation with the next nearest neighbor interactions, gives good agreement with the experimental values. In $\mathrm{sp}^{3} \mathrm{~d}^{5}$ orbital basis addition of the next nearest neighbor interactions for the anion gives correct explanation of the electronic band structure of semiconductors in its bulk as well as NCs [73]. Diaz et al.[74] have studied the electronic structure and optical spectra of GaAs NCs for a wide range of sizes by using both $s p^{3} s^{*}$ and $s p^{3} s^{*} d^{5}$ nearestneighbor TB models and found that the enclosure of $d$ orbitals into a minimal basis set is essential for a good explanation of the lowest lying states of electron, particularly in the well confined system. Franceschetti [56] has analyzed the electronic properties of PbSe NCs within the radius range of 8.4-14.8 $\AA$. Wannier function approach has been employed to study the band gap dependence on the different shapes (spherical, cubical, and tetrahedral) of CdS NCs and the report suggests that energy gap depends on the number of atoms present in the NC and not on their arrangements. Fig.9 shows the variation of energy gap variation as a function of the number of unit cells in the NC of different shapes, indicates that the energy gap slightly depends on the arrangements of the atoms in the NC [75].

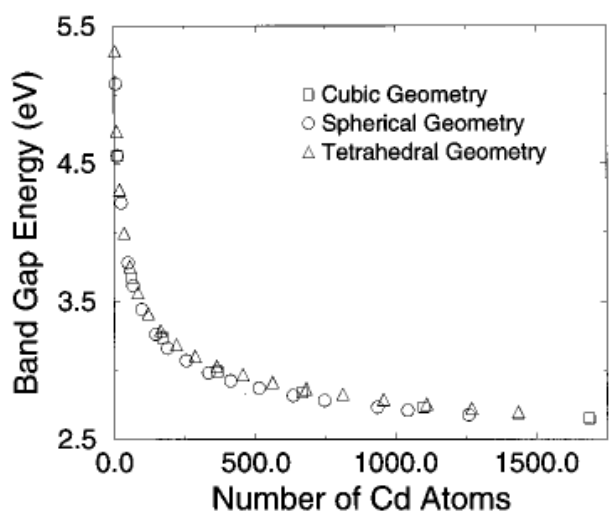

Figure 9. Dependence of band gaps on the number of Cd atoms for various shapes NC. Ref.[75].

Using first-principle DFT approach Srivastava et al. have analyzed the electronic properties of various bulk/NCs such as AlN, AlSb, AlAs [57-60] etc. in their different structural phases. The detailed discussion of the electronic structures of 1-D AIN NC in its various possible phases like zinc blende (B3), $\mathrm{NaCl}$ (B1) and $\mathrm{CsCl}$ (B2) is as follows. The band structure of $\mathrm{B} 3$ type phase of AIN NC as shown in Fig.10(a) has a band gap of about $1.6 \mathrm{eV}$ at $\Gamma$ point, 
defends the semiconducting nature. Fig.10(b) shows the band structure plot for B1 type phase of AlN NC, clearly depicts a band gap of around $1.5 \mathrm{eV}$ at $\Gamma$ point however the $\mathrm{B} 2$ type phase is purely metallic in nature. The band gaps of all the AlN 1-D NCs are quite smaller as compared to bulk crystal, which contradicts to the quantum size effect where usually enhancement in the band gaps has been observed. This abnormal AlN band gaps is due to the surface effects. The surface states related with the tri coordinated $\mathrm{N}$ and $\mathrm{Al}$ atoms at lateral facets in NC creates new energy levels at valence band edges as well as conduction band edges that directly narrows the band gaps relative to their bulk counterparts.
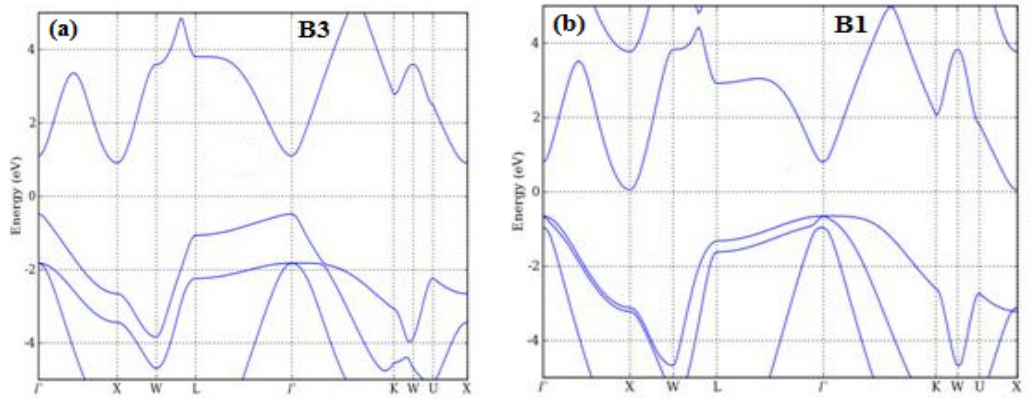

Figure 10. (a-b) Electronic Band structure of AIN NC in B3 and B1 type phase Ref.[60].

The density of states for the most stable B3 type phase of AlN NC has also been analyzed in the energy range of -5.0 to $5.0 \mathrm{eV}$ shown in Fig. 11, with three visible distinct peaks, where a highest peak appears in the valence band region near $-2.3 \mathrm{eV}$ and satisfies the band structure plot by showing the unavailability of peaks at the Fermi level.

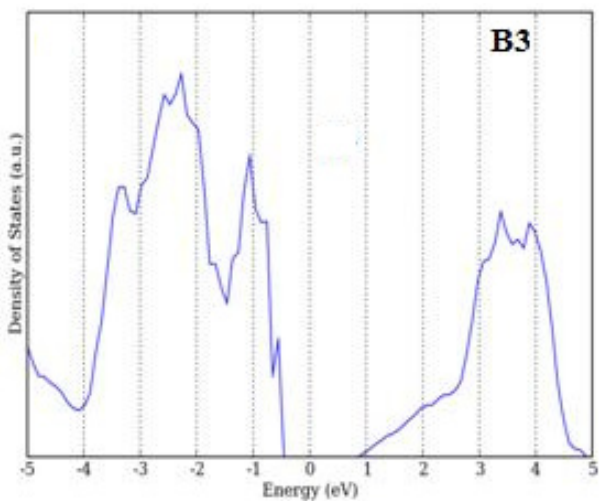

Figure 11. Density of states of AlN NC in different structural B3 phase Ref.[60].

\subsection{Optical properties}

It is well known that the band gaps of different semiconductors such as III-nitrides, II-VI group, IV group elements can be tuned by varying the composition in their alloys which 
make these systems prominent for various the applications like in optoelectronic devices, to use them for a spectral range starting from deep infrared to distant ultraviolet region. Quantum confinement has a great impact on the optical properties of semiconductor NCs. The phenomena of absorption and emission in the semiconductors are governed by their fundamental direct band gap. The increment in the energy band gap and strengthening of the oscillations with decrease in size of NCs has been revealed by spectroscopic studies. Due to the advancements in the synthesis, characterization and size selections of direct band structures of III-V and II-VI semiconductors, the spectroscopic studies of quantum confinement effects in these systems become much easier. The potential candidature of semiconductor NCs as light absorbers in solar-cell devices of third generation, has attracted number of researchers. For example PbSe, PbS, CdSe, PbTe, InAs and Si NCs shows much efficient multiple-exciton generation i.e. the chance of producing multiple electron-hole pairs from a single, high-energy photon, which thus increased the solar cell power [76]. For the solar energy conversion the semiconductor NCs requires a good match between the NC absorption spectrum and the solar spectrum. Keeping in mind that the quantum confinement effect tends to open up the energy band gap, the narrow band gap semiconductor NCs such as Ge, InAs, group-III antimonides and lead chalcogenides [77] are the potential candidates for the solar cell applications. Due to indirect band gap bulk $\mathrm{Si}$ is a poor light emitting material. In Si the excited electron-hole pairs have very wide radiative lifetime (ms), hence opposing non-radiative recombination rule and cause most of the excited electron-hole pairs to recombine non-radiatively. Si NCs formed by aerosol technique or by thermal precipitation of $\mathrm{Si}$ atoms rooted in $\mathrm{SiO}_{2}$ layers, give strong visible and near infrared photoluminescence (PL), similar characteristics as in porous Si [78]. Optical properties of isolated Si NCs of $\sim 4 \mathrm{~nm}$ size have been analysed with spectroscopic ellipsometry in the photon energy range of 1.1-5.0 eV. Si NCs reveals a major reduction in the dielectric functions and optical constants and whereas shows a large blue shift $(\sim 0.6 \mathrm{eV})$ in the absorption spectrum. The dielectric function and optical constants of the Si NC are shown in Fig. 12 and 13, where overall spectral features of Si NC are similar to bulk Si. Nevertheless, the Si NC shows a remarkable drop in the optical constants and dielectric function in contrast to bulk $\mathrm{Si}$. It is well recognized that decrease in the static dielectric constant becomes significant as the size of the structure approaches to the nano regime [79].

Photoluminescence (PL) properties of $\mathrm{GaAs} \mathrm{NCs}_{\text {in }} \mathrm{SiO}_{2}$ matrices formed by sequential ion implantation and thermal annealing, have been studied and observe broad PL due to GaAs NCs appears in the red spectral region, where the spectral shape of the red PL band depends on the hydrogen concentration in the sample. These $\mathrm{GaAs} \mathrm{NCs}$ in $\mathrm{SiO}_{2}$ film with low defects density will be used as the material for the future excitonic devices [80]. The blinking (or ON-OFF) behavior has been observed in single CdSe NC PL, which gets attributed to an intermittent photo ionization and subsequent neutralization of the NC [81]. The optical properties are related to the edge transition of the electronic band gaps in a semiconductor. Hence it is necessary to study the size dependence of the electronic band gap and the related exciton energy in semiconductor NCs. Few earlier experiments found that the lack of a large overlap between absorption and emission spectra in CdSe NC can improve the efficiency of light-emitting diodes (LEDs) due to the reduction in re-absorption 
[82]. Photovoltaic response has been investigated in PbSe NCs of various sizes and conjugated polymers. The devices composed with these NCs shows good diode characteristics and sizable photovoltaic response in a spectral range from the ultraviolet to infrared [83]. A very interesting observation about the NCs is that their band gap can be tuned over a large energy range simply via synthetic control over the size of the NCs [84]. NC having absorption onset in the near to mid infrared (IR) will also strongly absorb solar photons of higher energy. Generation of multiexcitons in PbSe NCs from a single photon absorption event is observed to take place in picosecond and occurs with up to $100 \%$ efficiency depending upon the excess energy of the absorbed photon. Thermodynamic conversion efficiency in solar cells is found to be $43.9 \%$ in concentrated solar lighting. The computed percentage depends upon the hypothesis that the absorption of an individual photon with energy above a semiconductor band gap results in formation of a single exciton and the photon energy which is more than the energy band gap get lost by electron-phonon interactions. Numerous techniques have existed to raise the power exchange efficiency of solar cells together with the improvement of multi-junction cells, intermediate band devices, hot electron extraction and carrier multiplication [85,86]. In Si35 and Si66 cores passivated with oxide shows lowering of energy band gap by $2.4 \mathrm{eV}$ and $1.5 \mathrm{eV}$. The oxide and hydrogen passivated NCs are optically forbidden indirect band gap and optically allowed direct band gap transitions respectively. However the highest occupied molecular orbitals and lowest unoccupied molecular orbitals are delocalized in both cases. Theoretical prediction get confirmed through the experimental observation that hydrogen passivated $\mathrm{Si}$ NCs emit blue light whereas oxide passivated Si NCs emit yellow-red light [87].

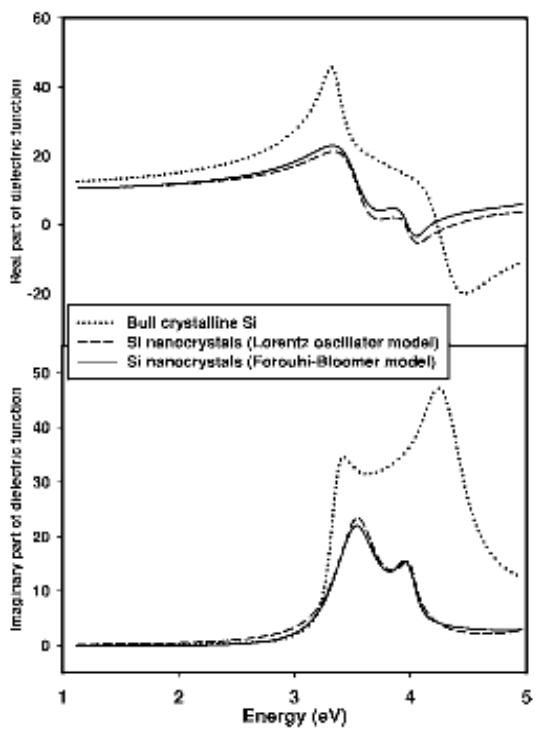

Figure 12. Real $(\varepsilon 1)$ and imaginary $(\varepsilon 2)$ parts of the complex dielectric function of the Si NC obtained from the spectral fittings based on the Lorentz oscillator model and the FB model. The dielectric function of bulk $\mathrm{Si}$ is also included for comparison Ref.[79]. 


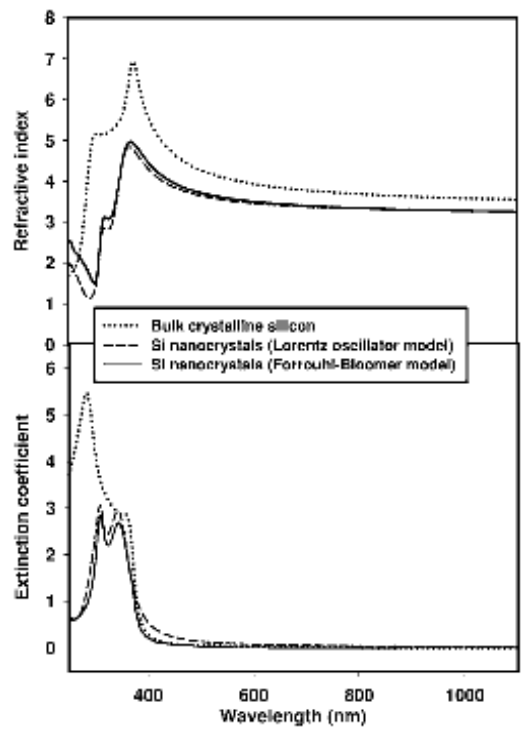

Figure 13. Refractive index (n) and extinction coefficient (k) of the Si NC and bulk Si as function of wavelength Ref.[79].

\subsection{Mechanical properties}

Mechanical properties of nano-materials are mainly focused on their constitutive response as well as on the fundamental physical mechanisms. Numbers of characteristics of mechanical behavior are presented to-date, like young modulus, shear modulus, bulk modulus, elastic constants and compressive strength etc. However literature shows that particularly in semiconducting NCs, these properties are yet not very much exploited; a very few reports are available on the bulk modulus of NCs which actually tells about the hardness of the material and inversely shows the compressibility of the same structure. The first-principles calculations using the LDA as exchange correlation functional, the variation in the bulk modulus of silicon nanocrystal have been investigated. On decreasing the size of the NC an enhancement in the bulk modulus has been observed [88]. In case of CdSe NCs structural transformation has been studied using high pressure X-ray diffraction and also calculates the bulk modulus $\left(\mathrm{B}_{0}\right)$, defined as the reciprocal of the volume compressibility, as \pm 74 GPa for rock salt phase of NCs and in close match with others report data for CdSe bulk and NCs. However due to the overlap of the numerous wurtzite diffraction peaks the exact value of $\mathrm{B}_{0}$ has not achieved [89]. Another example is GaN where its bulk and NCs have been studied using X-ray diffraction and calculated bulk moduli are reported as 187 and 319 GPa for the wurtzite phase of bulk and $\mathrm{NCs}$ respectively, although the respective $\mathrm{NaCl}$ phases are found to have very similar bulk moduli 208 and $206 \mathrm{GPa}$ [90]. A in-situ high-pressure study observes semiconductor to metal phase transitions in $\mathrm{ZnS}$ materials with average grain sizes of $10 \mu \mathrm{m}$ and $11 \mathrm{~nm}$. Bulk modulus and its pressure derivative $\left(\mathrm{B}_{0}{ }^{\prime}\right)$, of $\mathrm{B} 3$ phase has been estimated from the Birch-Murnaghan equation of state in a pressure range from 0 to $9 \mathrm{GPa}$ as $\mathrm{B}_{0}=72 \pm 7 \mathrm{GPa}$ and $\mathrm{B}_{0}{ }^{\prime}=9 \pm 3$ for $11 \mathrm{~nm}$ 
$\mathrm{ZnS}$ and $\mathrm{B}_{0}=68 \pm 3 \mathrm{GPa}$ and $\mathrm{B}_{0}{ }^{\prime}=7 \pm 1$ for $10 \mu \mathrm{m}$ ZnS NCs [91]. Another example is X-ray diffraction study of pressure-induced phase transformations in $\mathrm{ZnO}$ nanorods shows that the reduction of the particle size for $\mathrm{ZnO}$ crystallites leads to a significant increase of the bulk modulus and the pressure range of the coexistence of the wurtzite and rocksalt phases suggest that hardness of $\mathrm{ZnO}$ nanorods is higher than its bulk counterpart [92]. Raman study of nanocrystalline $\mathrm{ZnO}$ with different average crystalline sizes, predicts that the average elastic modulus of nanocrystalline $\mathrm{ZnO}$ shows a non-monotonic variation with crystalline size, suggests that the non-monotonic behavior is due to interplay between the elastic properties of the individual grains and those of the intergranular region [93]. A study on AlN NCs /nanowires reveals that the wurtzite structure of AIN NCs and nanowires have the $\mathrm{B}_{0} \sim 215.8$ GPa and $\sim 208.8 \mathrm{GPa}$, respectively, however the $\mathrm{B}_{0}$ of rocksalt phase of AlN NCs and nanowires are $\sim 312.6 \mathrm{GPa}$, and $324.9 \mathrm{GPa}$, respectively. This shows that the decrease of particle size can appreciably lead to an increase of the $\mathrm{B}_{0}[94]$.

\subsection{Magnetic properties}

Scientists divides the information technology into two parts one to exploits the degree of freedom of electronic charge in semiconductors to advances the information and other in magnetic materials where the spin degree of freedom is used to preserve the information. Spin of the charge carriers plays a slight role on semiconductor devices since most of the well known semiconductor devices are based on non-magnetic $\mathrm{Si}$ and GaAs which shows very less effect of spin. In contrast the superior spin related features have been acknowledged in magnetic semiconductors and diluted magnetic semiconductors (DMS) because of the coexistence of both magnetic and semiconductor properties. Nano magneto electronics is a newly developed field, where the two degrees of freedom, the charge and spin of the carriers, are utilized simultaneously to create new spintronics devices. $\mathrm{Mn}^{2+}$ or $\mathrm{Co}^{2+}$ the transition metal ions are the important dopants as they act as optically active impurities and localized spins in semiconductors and responsible for the optical and magnetic functionalities. Magnetic circular dichroism (MCD) spectroscopy of Mn doped and ZnS coated CdS NCs gives the electronic structures of Mn impurities and calculate the amount of Mn composition in NCs. Fig.14 shows the temperature dependence of the Zeeman splitting $\left(\Delta \mathrm{E}_{\mathrm{z}}\right)$ of $0.2,2$, and $10 \mathrm{~mol} \% \mathrm{Mn}$ doped CdS NCs, where the MCD peak signal is allotted to Zeeman splitting of the lowest exciton. The study reveals that MCD signal directly depends on the Zeeman splitting and the first derivative of the absorption spectrum, though the Zeeman splitting is smaller than the bandwidth of the lowest exciton absorption [95].

Magnetic ion doped semiconductors are called diluted magnetic semiconductors (DMSs) [96] and these DMSs are expected to be a key material for spintronics devices due to their spindependent effects [97]. According to the literature not much report are available on the magnetic properties of semiconductor NCs. However, Magnetic properties of ZnS and ZnO NCs doped with various concentrations of a transition metals have been analyzed. Over a wide range of dopant concentrations without changing the size (1.6 and $4.7 \mathrm{~nm}$ ) of the $\mathrm{NC}$, the study clearly reveals the magnetic properties of such doped systems, remaining paramagnetic down to the lowest temperature, can give precise information concerning the dopant level in 
such samples [98,99]. ZnO NC with Co doping and without capping agent shows ferromagnetic and paramagnetic behavior, respectively. Co intra $\mathrm{d}-\mathrm{d}$ transition raises in ferromagnetic NCs which may be because of the profound bound states at the NCs surface. Fig. 15 shows the variation of magnetization as a function of magnetic field for Co doped $\mathrm{ZnO}$ NCs and in case of washed NCs (also called as-prepared) the magnetization is directly proportional to the applied magnetic field lacking hysteresis, which shows paramagnetic behavior. However the ferromagnetic nature with hysteresis has been seen in the $\mathrm{O}$ capped NCs sample. As the high magnetic field increases the paramagnetic behavior is also predicted, which indicates that paramagnetic and ferromagnetic NCs coexist in these samples [100].

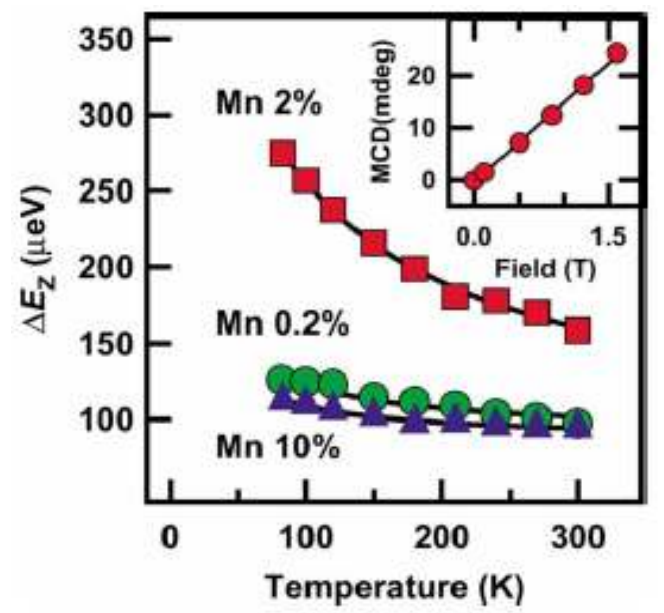

Figure 14. (a) Dependence of the Zeeman splitting energy on Temperature of CdS NCs doped with 0.2, 2 , and $10 \mathrm{~mol} \% \mathrm{Mn}$ ions. The inset shows the magnetic field dependence of the MCD signal intensity in $2 \mathrm{~mol} \%$ Mn doped CdS NCs Ref.[95].

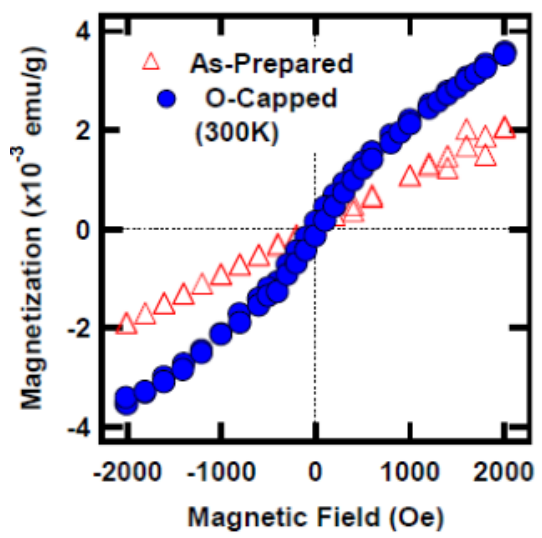

Figure 15. Variation of magnetization with respect to magnetic field at $300 \mathrm{~K}$ of Co doped $\mathrm{ZnO}$ NCs Ref.[100]. 
Mn doped GaN NCs with the average diameters ranging from 4-18 nm has been prepared at low temperature and reveals that the NCs exhibit ferromagnetism with magnetization and Curie temperature values increasing with the concentration of $\mathrm{Mn}$ and particle size [101]. The magnetic properties of hydrogenated Si NCs doped with pairs of Mn atoms were investigated using spin density functional theory, where the two sites occupied by Mn, shows strong dependence of obtained formation energies and total magnetic moments. Pairs at sites with the same character tend to ferromagnetic spin arrangements, significantly influenced by their noncollinearity [102]. As a theoretical understanding one Ab-initio pseudopotentials approach has been employed to investigate the electronic and magnetic properties of Mn doped Ge, GaAs, and ZnSe NCs. To predict that the ferromagnetic and half-metallicity trends found in the bulk are preserved in the NCs. Due to localization of Mn states, they are less affected by quantum confinement. As a result in NCs, the Mn-related impurity states become much deeper in the gap with decreasing size [103].

\subsection{Structural phase transition}

The effects of crystal size on the stability of NC is of considerable interest because for utilizing a material in several applications, information about crystal structure at that particular temperature and pressure is must. In nano-materials the reduction in the size of the crystal changes its entire properties; this may be because of the change in the lattice parameters for a given phase and also due to pressure effects. If we assume a spherical NC, then pressure applied by the surface on the core of the NC will lead to the relaxation of the first atomic planes and therefore to an increase or a decrease in the lattice parameter. This effect is well seen in semiconductors, due to the adsorption state of the surface and surface itself reconstructs themselves to minimize the number of dangling bonds, which produces more stresses in the upper layers. Observation shows that in NC, these stresses will also contribute to the surface pressure and to the reduction or extension of atomic bonds. One obvious question arises that how will the relative stability of different possible solid structures change for NCs with respect to bulk materials? One way to answer this question is to use the pressure to force nanostructured materials to get convert it from one solid structure to another. It has been known classically that when pressure is applied the structure of the solid changes and hence its density as well as volume, that leads to the overlapping of the electron shells and structural phase transformation may take place. In III-V compound semiconductors the zinc blende to rocksalt phase transition are generally expected and intermediate phase NiAs are also seen sometimes and further the structure stabilized in a high pressure $\mathrm{CsCl}$ phase. The efforts on the high pressure behavior of NC starts from the year 1994 by S. H. Tolbert and A. P. Alivisatos [104] as both are the pioneer workers in the field of high pressure structural transformation in NCs. They discussed the stability of CdSe NCs in wurtzite and rocksalt phases and observed the phase transition in the range of 3.6 to $4.9 \mathrm{GPa}$ for crystallites radius ranging from 21 to $10 \AA$. There are also some reports on the high pressure behaviour of Si and CdS NCs, shows that the smaller the crystallite higher the transition pressure, which has been explained by them in terms of surface energy differences between the phases involved [105,106]. Qadri et al. [107] 
reported that the effect of reduced grain size in PbS NCs elevates the transition pressure, while the compressibility increases with decreasing grain size. An enhancement of transition pressure has been observed in ZnO NCs [108], ZnS [5] and PbS [6] in comparison to its bulk counterpart. In an another report the pressure induced I-II transition has been studied for nanocrystalline Ge of size 13, 49 and $100 \mathrm{~nm}$ using synchrotron x-ray diffraction, where the bulk modulus and the transition pressure increases with decrease in particle size for both Ge-I and Ge-II, but the percentage volume collapse at the transition remains constant [109]. The hexagonal AlN nanocrystals have a particle size of $\sim 10 \mathrm{~nm}$ (Fig. 16) shows wurtzite to rocksalt phase transition at around $14.5 \mathrm{GPa}$, which is significantly lower than the transition pressure of $22.9 \mathrm{GPa}$ observed for the bulk AlN by using the same technique. Fig.17 clearly illustrates that at the phase transition pressure of $14.5 \mathrm{GPa}$ the high-pressure rocksalt phase is about $20.5 \%$ denser than the hexagonal wurtzite phase [110].

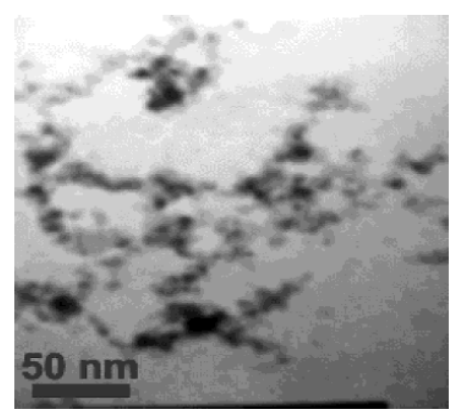

Figure 16. TEM image of AlN NCs Ref.[110].

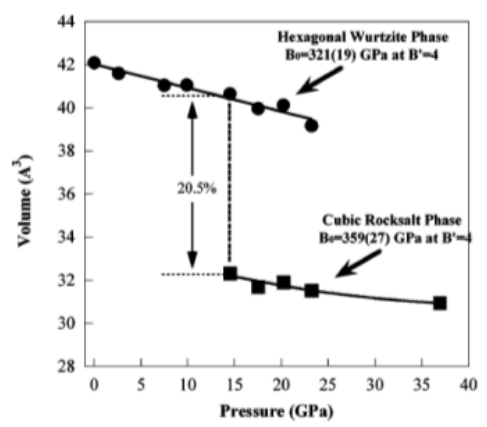

Figure 17. Equation of state plot and the relative volumetric variation of $\mathrm{AlN}$ upon the phase transition at $14.5 \mathrm{GPa}$.

Wen et al. [111] have studied the pressure induced phase transition in CdSe and ZnO NCs and found that the transition pressure increases as the nanocrystals size reduces. A pressure induced first-order structural phase transition from wurtzite to rocksalt type structure has been observed in GaN NCs at around $48.8 \mathrm{GPa}$ using x-ray diffraction technique [112]. Theoretically [58] the structural stability and high pressure behavior of AlAs NCs has been 
analysed in various phases such as wurtzite (B4), zincblende (B3), $\mathrm{CsCl}$ (B2) and $\mathrm{NaCl}$ (B1), and observes the structural transformations from $\mathrm{B} 3 \rightarrow \mathrm{B} 1$ at around $8.9 \mathrm{GPa}, \mathrm{B} 3 \rightarrow \mathrm{B} 2$ at 7.12 $\mathrm{GPa}$ and from $\mathrm{B} 3 \rightarrow \mathrm{B} 4$ at $3.88 \mathrm{GPa}$, which is probably being the first report. The calculated values of transition pressures for AlAs NCs are lower in comparison to its bulk counterpart $[57,113,114]$. The amount of volume collapse at the transition pressure has also been analyzed and the computed volume collapse for B3 $\rightarrow \mathrm{B} 4, \mathrm{~B} 3 \rightarrow \mathrm{B} 2$ and $\mathrm{B} 3 \rightarrow \mathrm{B} 1$ transitions are found to be $5.8 \%, 3.5 \%$ and $1.5 \%$ respectively as illustrated in Fig. 18 .

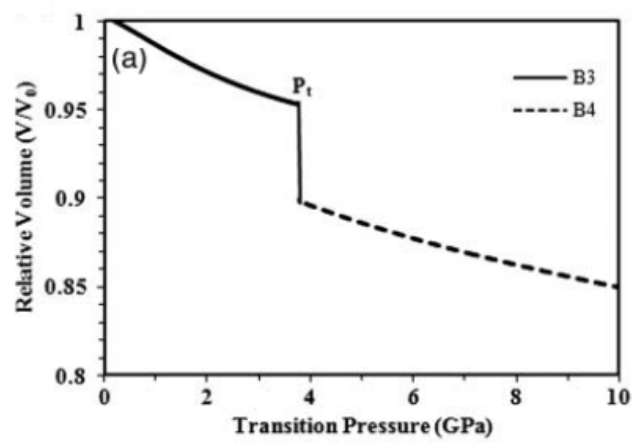

Figure 18. Relative volume as a function transition pressure for AlAs NCs Ref.[58].

Similar approach has been employed to investigate the structural stability of $\sim 1 \mathrm{~nm}$ sized $\mathrm{AlSb} \mathrm{NC}$ [58] in its B3, B1 and B2 type phases under high compression and confirms that the B3 type phase is the most stable amongst the other considered phases, further under compression, the original B3 type phase of AlSb NC transforms to B1 type phase at a pressure of about $8.9 \mathrm{GPa}$, which is comparatively larger than that of bulk crystal. This discussion concludes that the transition pressure strictly depends on the size of the NC and may vary accordingly.

\section{Conclusion}

In this chapter we have described four topics; first, we have explained the fundamentals behind the peculiar properties of semiconductor NCs, the factors that makes them different from their bulk counterparts. Second, recent applications of NCs in electronic, optoelectronics, photonics as well as in medicines have been discussed. Third, we referred the present synthesis and characterization techniques for these tiny crystals, discussed various recent methods of controlled synthesis on growth and size of NCs. Fourth, we have remarked on the various properties of semiconductor NCs such as structural, electronic, optical, mechanical, magnetic properties of NCs with a detailed description of the structural transformations of semiconductor NCs under the application of pressure. We believe that the discussion on semiconductor NCs with recent results and ideas will be certainly be helpful, especially to variety of scientific community like physicists, chemists, and biologists, nano researchers/scientists for the advancements of science and technology, and its exploitation in variety of applications. 


\section{Author details}

Anurag Srivastava and Neha Tyagi

Advance Material Research Group, Computational Nanoscience and Technology Laboratory, $A B V$ - Indian Institute of Information Technology and Management, Gwalior (M.P.), India

\section{Acknowledgement}

Authors are grateful to ABV-Indian Institute of Information Technology and Management, Gwalior for providing the infrastructural support. One of us Neha Tyagi is thankful to ABVIIITM for the award of Ph.D scholarship.

\section{References}

[1] Achermann M., Petruska M. A., Kos S., Smith D. L., Koleske D. D., Ekimov V. I. Energytransfer pumping of semiconductor nanocrystals using an epitaxial quantumwell. Nature 2004; 429,642-646.

[2] Huynh W. U., Dittmer J. J., Alivisatos A. P. Hybrid Nanorod-Polymer Solar Cells. Science 2002; 295(5564) 2425-2427.

[3] Klein D. L., Roth R., Lim A. K. L., Alivisatos A. P., McEuen P. L. A single-electron transistor made from a cadmium selenide nanocrystal. Nature 1997;389(6652)699-701.

[4] Boinovich L.B., Emelyanenko A. M. Hydrophobic materials and coatings: principles of design, properties and applications. Russian Chemical Reviews 2008;77(7)583-600.

[5] Jiang J. Z., Gerward L., Frost D., Secco R., Peyronneacu J., Olsen J. S. Grain-size effect on pressure-induced semiconductor-to-metal transition in ZnS. Journal of Applied Physics 1999;86(11)6608-6610.

[6] Jiang J. Z., Gerward L., Frost D., Secco R., Peyronneacu J., Olsen J. S., Truckenbrodt J. Phase transformation and conductivity in nanocrystal $\mathrm{PbS}$ under pressure. Journal of Applied Physics 2000;87(5) 2658-2660.

[7] Shiang J.J., Kadavanich A.V., Grubbs R.K., Alivisatos A.P. Symmetry of Annealed Wurtzite CdSe Nanocrystals: Assignment to the C-3v Point Group. Journal of Physical Chemistry 1995;99(48)17417-17422.

[8] Colvin V. L., Schlamp M. C., Alivisatos A. P. Light-emitting diodes made from cadmium selenide nanocrystals and a semiconducting polymer. Nature 1994;370(6488) 354-357.

[9] Peng X., Manna L., Yang W., Wickham J., Scher E., Kadavanich A., Alivisatos A. P. Shape control of CdSe nanocrystals. Nature 2000;404(6773) 59-61.

[10] Achermann M., Petruska M. A., Koleske D. D., Crawford M. H., Klimov V. I. Nanocrystal-based light-emitting diodes utilizing high-efficiency nonradiative energy transfer for color conversion. Nano Letters 2006;6(7)1396-1400.

[11] Tessler N., Medvedev V., Kazes M., Kan S.H., Banin U. Efficient Near-Infrared Polymer Nanocrystal Light-Emitting Diodes. Science 2002;295(5559) 1506-1508.

[12] Mueller A.H., Petruska M.A., Achermann M., Werder D.J., Akhadov E.A., Koleske D.D., Hoffbauer M.A., Klimov V.I. Multicolor light-emitting diodes based on semiconductor nanocrystals encapsulated in GaN charge injection layers. Nano Letters 2005;5(6)1039-1044. 
[13] Peralvarez M., Barreto J., Carreras J., Morales A., Urrios D. N., Lebour Y., Dominguez C., Garrido B. Si-nanocrystal-based LEDs fabricated by ion implantation and plasmaenhanced chemical vapour deposition. Nanotechnology 2009;20(405201)1-10.

[14] Gudiksen M.S., Maher K. N., Ouyang L., Park Hongkun. Electroluminescence from a Single-Nanocrystal Transistor, Nano Letters 2005;5(11) 2257-2261.

[15] Kahng D., Sze S. M. A floating-gate and its application to memory devices, The Bell System Technical Journal 1967;46(4) 1288-1295.

[16] Tiwari S., Rana F., Hanafi H., Hartstein A., Crabbe E.F., Chan K. A silicon nanocrystals based memory. Applied Physics Letters 1996;68(10) 1377-1379

[17] Chang T. C., Jian F. Y., Chen S. C., Tsai Y. T. Development in nanocrystal memory. Materials today 2011;14(12) 608-615.

[18] Ammendola G., Ancarani V., Triolo V., Bileci M., Corso D. , Crupi I. , Perniola L., Gerardi C., Lombardo S., DeSalvo B. Nanocrystals memories for FLASH device applications. Solid-State Electronics 2004;48 (9)1483-1488.

[19] Kim J. K., Cheong H. J., Kim Y., Yi J. Y., Bark H. J., Bang S. H. and Cho J. H. Rapidthermal-annealing effect on lateral charge loss in metal-oxide-semiconductor capacitors with Ge nanocrystals. Applied Physics Letters 2003;82(15) 2527-2529.

[20] Lu X. B., Lee P. F., Dai J. Y. Synthesis and memory effect study of Ge nanocrystals embedded in $\mathrm{LaAlO}_{3}$ high-k dielectrics. Applied Physics Letters 2005;86(203111) 1-3.

[21] Du H., Chen C., Krishnan R., Krauss T. D., Harbold J. M., Wise F. W., Thomas M. G., Silcox J. Optical Properties of Colloidal PbSe Nanocrystals. Nano Letters 2002;2(11)13211324.

[22] Choudhury K. R., Sahoo Y., Ohulchanskyy T. Y., Prasad P. N. Efficient photoconductive devices at infrared wavelengths using quantum dot-polymer nanocomposites. Applied Physics Letters 2005;87 (7) 073110-1 073110-3.

[23] Qi D., Fischbein M., Drndic M., Selmic S. Efficient polymer-nanocrystal quantum-dot photodetectors. Applied Physics Letters 2005;86 (9) 093103-1 093103-3.

[24] Ma X., Yuan B., Yan Z. The photodetector of Ge nanocrystals/Si for $1.55 \mu \mathrm{m}$ operation deposited by pulsed laser deposition, Optics Communications 2006;260(1) 337-339.

[25] Guimard D., Morihara R., Bordel D., Tanabe K., Wakayama Y., Nishioka M., Arakawa Y. Fabrication of InAs/GaAs quantum dot solar cells with enhanced photocurrent and without degradation of open circuit voltage. Applied Physics Letters 2010;96 (20) 203507-1 203507-3.

[26] Gur I., Fromer N.A., Geier M. L., Alivisatos A. P. Air-Stable All-Inorganic Nanocrystal Solar Cells Processed from Solution. Science 2005;310(5747) 462-465.

[27] Gross N. Y., Harari M. S., Zimin M., Kababya S., Schmidt A., Tessler N. Molecular control of quantum-dot internal electric field and its application to CdSe-based solar cells. Nature Materials 2011;10(12) 974-979.

[28] Svrcek V., Mariotti D., Nagai T., Shibata Y., Turkevych I., Kondo M. Photovoltaic Applications of Silicon Nanocrystal Based Nanostructures Induced by Nanosecond Laser Fragmentation in Liquid Media. Journal of Physical Chemistry C 2011;115(12)5084-5093.

[29] Katteboinaa Suman, V S R Chandrasekhar. P, Balaji S. Drug Nanocrystals: a Novel Formulation Approach for Poorly Soluble Drugs. International Journal of PharmTech Research 2009;1(3)682-694 (2009). 
[30] Liversidge E. M. M., Liversidge G. G. Drug Nanoparticles: Formulating Poorly WaterSoluble Compounds. Toxicologic Pathology 2008;36(1)43-48.

[31] Tran P.T., Goldman E.R., Anderson G.P., Mauro J.M., Mattoussi H. Use of luminescent CdSe-ZnS nanocrystal bioconjugates in quantum dot-based nanosensors. Physica Status Solidi B 2002;229(1), 427-432.

[32] Dubertret B., Skourides P., Norris D. J., Noireaux V., Brivanlou A. H., Libchaber A. In vivo imagining of quantum dots encapsulated in phospholipid micelles. Science 2002;298(5599) 1759-1762.

[33] Parak W. J., Boudreau R., Gros M. L., Gerion D., Zanchet D., Micheel C. M., Williams S. C., Alivisatos A. P., Larabell C. Cell motility and metastatic potential studies based on quantum dot imaging of phagokinetic tracks. Advanced Materials 2002;14(12) 882-885.

[34] Taylor J. R., Fang M. M., Nie S. M. Probing specific sequences on single DNA molecules with bioconjugated fluorescent nanoparticales. Analytical Chemistry 2000;72(9)19791986.

[35] Parak W. J., Pellegrino T., Plank C. Labelling of cells with quantum dots. Nanotechnology 2005;16(2) R9-R25.

[36] Brus L. Electronic wave functions in semiconductor clusters: experiment and theory. Journal of Physical Chemistry 1986;90(12)2555-2560.

[37] Steigerwald M. L., Alivisatos A. P., Gibson J. M., Harris T. D., Kortan R., Muller A. J., Thayer A. M., Duncan T. M., Douglass D. C. and Brus L. E. Surface derivatization and isolation of semiconductor cluster molecules. Journal of American Chemical Society 1988;110(10)3046-3050.

[38] Yong K.-T., Sahoo Y., Swihart M. T., Prasad P. N. Shape Control of CdS Nanocrystals in One-Pot Synthesis. Journal of Physical Chemistry C 2007;111(6) 2447-2458.

[39] Wang W., Poudel B., Huang J. Y., Wang D. Z., Kunwar S., Ren Z. F. Synthesis of gramscale germanium nanocrystals by a low-temperature inverse micelle solvothermal route. Nanotechnology 2005;16(8)1126-1129.

[40] Chen L., Holmes J. D., Garcia S. R., Morris M. A. Facile Synthesis of Monodisperse ZnO Nanocrystals by Direct Liquid Phase Precipitation. Journal of Nanomaterials 2011; 2011, 1-9.

[41] Yu M., Hao X., Cui D., Wang Q., Xu X., Jiang M. Synthesis of aluminium nitride nanocrystals and their catalytic effect on the polymerization of benzene. Nanotechnology 2003;14(1) 29.

[42] Yang H., Yao X., Huang D. Sol gel synthesis and photoluminescence of AlP nanocrystals embedded in silica glasses. Optical Materials 2007;29(7)747-752.

[43] Yang H.Q., Yao X., Huang D.M., Wang X. J., Shi H. Z., Zhang B. L., Liu S. X., Fang Y. Sol-gel synthesis and photoluminescence of III-V semiconductor InAs nanocrystals embedded in silica glasses. Journal of Nanoscience and Nanotechnology 2005;5(5)786-9.

[44] Yang H., Huang D., Wang X., Gu X., Wang Fujian, Xie S., Yao X. Sol-Gel Synthesis of Luminesent InP Nanocrystals Embedded in Silica Glasses. Journal of Nanoscience and Nanotechnology 2005;5 (10)1737-1740.

[45] Nayak J., Mythili R., Vijayalakshmi M., Sahu S. N. Size quantization effect in GaAs nanocrystals. Physica E: Low-dimensional Systems and Nanostructures 2004; 24(34)227-233. 
[46] Yuk J. M., Kim K., Lee Z., Watanabe M., Zettl A., Kim T. W., No Y. S., Choi W. K., Lee J. Y. Direct fabrication of zero- and one-dimensional metal nanocrystals by thermally assisted electromigration. ACS Nano 2010;4(6)2999-3004.

[47] Chiu C. W., Liao T. W., Tsai K. Y., Wang F. M., Suen Y. W., Kuan C. H. Fabrication method of high-quality Ge nanocrystals on patterned Si substrates by local melting point control. Nanotechnology 2011;22(27)275604, 1-5.

[48] Namatsu H., Kurihara K., Nagase M., Makino T. Fabrication of 2-nm-wide silicon quantum wires through a combination of a partially-shifted resist pattern and orientation-dependent etching. Applied Physics Letters 1997;70(5) 619-621.

[49] Murray C. B., Norris D. J., Bawendi M. G. Synthesis and characterization of nearly monodisperse CdE (E = sulfur, selenium, tellurium) semiconductor nanocrystallites. Journal of American Chemical Society 1993;115(19) 8706-8715.

[50] McCaldin J. O. Current approaches to p-n junction in wider band gap II-VI semiconductors. Journal of Vaccum Science and Technology A 1990;8(2), 1188-1193.

[51] Alphandery E., Nicholas R. J., Mason N. J., Lyapin S. G., Klipstein P. C. Photoluminescence of self-assembled InSb quantum dots grown on $\mathrm{GaSb}$ as a function of excitation power, temperature, and magnetic field. Physical Review B 2002;65,115322-1 115322-7.

[52] Xie Y., Qian Y.T., Wang W.Z., Zhang S.Y., Zhang Y.H. A Benzene-Thermal Synthetic Route to Nanocrystalline GaN. Science 1996;272(5270)1926-1927.

[53] Dvorak M.D., Justus B.L., Gaskill D. K., Hendershot D.G. Nonlinear absorption and refraction of quantum confined InP nanocrystals grown in porous glass. Applied Physics Letters 1995;66(7) 804-806.

[54] Kim S. S., Bang K.I., Kwak J., Lim K. S. Growth of Silicon Nanocrystals by LowTemperature Photo Chemical Vapor Deposition. Japanese Journal of Applied Physics 2006;45(1)L46-L49.

[55] Costales A., Blanco M. A., Francisco E., Solano C. J. F., Pendas A. M. Theoretical Simulation of AlN Nanocrystals. Journal of Physical Chemistry C 2008;112(17) 6667-6676.

[56] Franceschetti A. Structural and electronic properties of PbSe nanocrystals from first principles. Physical Review B 2008;78,075418-1 075418-6.

[57] Srivastava A., Tyagi N., Singh R.K. Pressure induced phase transformation and electronic properties of AlAs. Materials Chemistry Physics 2011;125(1-2)66-71.

[58] Srivastava A., Tyagi N. High pressure behavior of AlAs nanocrystals: the first-principle study. High Pressure Research iFirst 2012, 1-5.

[59] Tyagi N., Srivastava A. Structural Phase Transition and Electronic Properties of AlSb Nanocrystal. Proc. Intl. Conf. on Nanosci. Eng. Tech. (ICONSET 2011) 978-1-4673-00735/11/\$26.00@2011 IEEE IEEEXplore 2011, 421-423.

[60] Tyagi N., Srivastava A. Electronic properties of AlN nanocrystal: A first principle study. XVI National Seminar on Ferroelectrics and Dielectrics and (NSFD-XVI), American Institute of Physics Conference Proc. 2011;1372, 252-262.

[61] Z.-G., Bai Y.-J., Cui D.-L., Hao X.-P., Wang L.-M., Wang Q.-L., Xu X.-G. Increase of lattice constant in GaP nanocrystals. Journal of Crystal Growth 2002;242(3-4) 486-490.

[62] Efros Al. L., Efros A. L. Interband absorption of light in a semiconductor sphere. Soviet Physics Semiconductors 1982;16(7) 772-775. 
[63] Buda F., Kohanoff J., Parrinello M. Optical properties of porous silicon: A first-principle study. Physical Review Letters 1992;69(8) 1272-1275.

[64] Krishna M. V. R., Friesner R. A. Exciton spectra of semiconductor clusters. Physical Review Letters 1991;67(5) 629-632.

[65] Jancu J. M., Scholz R., Beltram F., Bassani F. Empirical spds* tight-binding calculations for cubic semiconductors: General method and materials parameters. Physical Review B 1998;57(11)6493-6507.

[66] Einevoll G.T. Confinement of excitons in quantum dots. Physical Review B 1992;45(7) 3410-3417.

[67] Nair S.V., Ramaniah L.M., Rustagi K.C. Electron states in a quantum dot in an effectivebond-orbital model. Physical Review B 1992;45(11) 5969-5979.

[68] Buuren T. V., Dinh L.N., Chase L.L., Siekhaus W.J., Terminello L.J. Changes in the Electronic Properties of Si Nanocrystals as a Function of Particle Size. Physical Review Letters 1998;80(17) 3803-3806.

[69] Wang L. W., Zunger A. Local-density-derived semiempirical pseudopotentials. Physical Review B 1995;51(24) 17398-17416.

[70] Fu H., Zunger A. Local-density-derived semiempirical nonlocal pseudopotentials for InP with applications to large quantum dots. Physical Review B 1997;55(3)1642-1653.

[71] Lippens P.E., Lannoo M. Calculation of the band gap for small CdS and ZnS crystallites. Physical Review B 1989;39(15)10935-10942.

[72] Sapra S., Viswanatha R., Sarma D. D. An accurate description of quantum size effects in InP nanocrystallites over a wide range of sizes. Journal of Physics D: Applied Physics 2003;36(13)1595-9.

[73] Sapra S., Shanthi N., Sarma D. D. Realistic tight-binding model for the electronic structure of II-VI semiconductors. Physical Review B 2002;66(8)205202-1 205202-8.

[74] Diaz J. G., Bryant G. W. Electronic and optical fine structure of GaAs nanocrystals: The role of d orbitals in a tight-binding approach. Physical Review B 2006;73(7) 075329-1 075329-9.

[75] Mizel Ari, Cohen M. L. Electronic energy levels in semiconductor nanocrystals: A Wannier function approach. Physical Review B 1997;56(11)6737-6741.

[76] Beard M. C., Knutsen K. P., Yu P., Luther J. M., Song Q., Metzger W. K., Ellingson R. J., Nozik A. J. Multiple exciton generation in colloidal silicon nanocrystals. Nano Letters 2007;7(8)2506-2512.

[77] Schaller R. D., Pietryga J. M., Goupalov S. V., Petruska M. A., Ivanov S. A., Klimov V. I. Breaking the Phonon Bottleneck in Semiconductor Nanocrystals via Multiphonon Emission Induced by Intrinsic Nonadiabatic Interactions. Physical Review Letters 2005;95(19)196401-1 196401-4.

[78] Cullis A. G., Canham L. T., Calcott P. D. J. The structural and luminescence properties of porous silicon. Journal of Applied Physics 1997;82(3)909-965.

[79] Ding L., Chen T. P., Liu Y., Ng C. Y., Fung S. Optical properties of silicon nanocrystals embedded in a $\mathrm{SiO}_{2}$ matrix. Physical Review B 2005;72(12)125419,1-7.

[80] Kanemitsu Y., Tanaka H., Kushida T., Min K.S., Atwater H. A. Luminescence properties of GaAs nanocrystals fabricated by sequential ion implantation. Journal of Luminesence 2000;87-89,432-434. 
[81] Nirmal M., Dabbousi B. O., Bawendi M. G., Macklin J. J., Trautman J. K., Harris T. D., Brus L. E. Fluorescence intermittency in single cadmium selenide nanocrystals. Nature 1996;383(6603)802-804.

[82] Schlamp M. C., Peng X., Alivisatos A. P. Improved efficiencies in light emitting diodes made with $\mathrm{CdSe}(\mathrm{CdS})$ core/shell type nanocrystals and a semiconducting polymer. Journal of Applied Physics 1997;82(11)5837-5842.

[83] Jiang X., Schaller R. D., Lee S. B., Pietryga J. M., Klimov V. I., Zakhidov A. A. PbSe nanocrystal/conducting polymer solar cells with an infrared response to 2 micron, Journal Materials Research 2007;22(8) 2204-2210.

[84] Schaller R. D., Klimov V. I. High Efficiency Carrier Multiplication in PbSe Nanocrystals: Implications for Solar Energy Conversion. Physical Review Letters 2004;92(18)186601,1-4.

[85] Green M. A. Third generation photovoltaics: solar cells for 2020 and beyond. Physica E 2002;14(1-2) 65-70.

[86] Nozik A. J. Spectroscopy and hot electron relaxation dynamics in semiconductor quantumwells and quantum dots. Annual Review of Physical Chemistry 2001;52, 193-231.

[87] Zhou Z., Brus L., Friesner R. Electronic Structure and Luminescence of 1.1- and 1.4-nm Silicon Nanocrystals: Oxide Shell versus Hydrogen Passivation. Nano Letters 2003;3(2)163-167.

[88] Cherian R., Gerard C., Mahadevan P., Cuong N. T., Maezono R. Size dependence of the bulk modulus of semiconductor nanocrystals from first-principles calculations. Physical Review B 2010;82(23) 235321, 1-7.

[89] Tolbert S. H., Alivisatos A. P. The wurtzite to rock salt structural transformation in CdSe nanocrystals under high pressure. Journal of Chemical Physics 1995;102(11)4642-4656.

[90] Jorgensen J.-E., Jakobsen J. M., Jiang J. Z., Gerward L., Olsen J. S. High-pressure X-ray diffraction study of bulk- and nanocrystalline GaN. Journal of Applied Crystallography 2003;36(2) 920-925.

[91] Jiang J. Z. Phase transformations in nanocrystals. Journal of Materials Science 2004;39(16-17)5103- 5110.

[92] Wu X., Wu Z., Guoc L., Liu C., Liu J., Li X., Xu H. Pressure-induced phase transformation in controlled shape $\mathrm{ZnO}$ nanorods. Solid State Communications 2005;135(11-12)780-784.

[93] Panchal V., Ghosh S., Gohil S., Kulkarni N., Ayyub P. Non-monotonic size dependence of the elastic modulus of nanocrystalline $\mathrm{ZnO}$ embedded in a nanocrystalline silver matrix. Journal of Physics: Condensed Matter 2008;20(34)345224,1-4.

[94] Lei W. W., Liu D., Zhang J., Cui Q. L., Zou G. T. Comparative studies of structural transition between AIN nanocrystals and nanowires. Journal of Physics: Conference Series 2008;121,162006,1-8.

[95] Taguchi S., Ishizumi A., Tayagaki T., Kanemitsu Y. Mn-Mn couplings in Mn-doped CdS nanocrystals studied by magnetic circular dichroism spectroscopy. Applied Physics Letters 2009;94(17)173101-173103.

[96] Ando K., Takahashi K., Okuda T., Umehara M. Magnetic circular dichroism of zincblende-phase MnTe. Physical Review B 1992;46(19)12289-12297.

[97] Furdyna J. K. Diluted magnetis semiconductor. Journal of Applied Physics 1988;64(4) R29-R64. 
[98] Wolf S. A., Awschalom A. A., Buhrman R. A., Daughton J. M., Molnar S., Roukes M. L., Chtchelkanova A. Y., Treger D. M. Spintronics: A Spin-Based Electronics Vision for the Future. Science 2001;294(5546)1488-1495.

[99] Sarma D.D., Viswanatha R., Sapra S., Prakash A., Hernandez M. G. Magnetic Properties of Doped II-VI Semiconductor Nanocrystals. Journal of Nanoscience and Nanotechnology 2005;5(9)1503-1508.

[100] Taguchi S., Tayagaki T., Kanemitsu Y. Luminescence and magnetic properties of Co doped ZnO nanocrystals. IOP Conf. Series: Materials Science and Engineering 2009;6, 012029, 1-4.

[101] Biswas K., Sardar K.,Rao C. N. R. Ferromagnetism in Mn-doped GaN nanocrystals prepared solvothermally at low temperatures. Applied Physics Letters 2006;89(13) 132503-132505.

[102] Panse C., Leitsmann R., Bechstedt F. Magnetic interaction in pairwise Mn-doped Si nanocrystals. Physical Review B 2010;82(12)125205, 1-9.

[103] Huang X., Makmal A., Chelikowsky J. R., Kronik L. Size dependent spintronics properties of diluted magnetic semiconductors nanocrystals. Physical Review Letters 2005;94(23)236801, 1-4.

[104] Tolbert S. H., Alivisatos A. P. Size Dependence of a First Order Solid-Solid Phase Transition: The Wurtzite to Rock Salt Transformation in CdSe Nanocrystals. Science 1994;265(5170)373-376.

[105] Tolbert S. H., Alivisatos A.P. The Wurtzite to Rock-Salt Structural Transformation in Cdse Nanocrystals under High-Pressure. Journal of Chemical Physics 1995;102(11)4642-4656.

[106] Tolbert S. H., Herhold A. B., Brus L. E., Alivisatos A. P. Pressure-induced structural transformations in Si nanocrystals: Surface and shape effects. Physical Review Letters 1996;76(23)4384-4387.

[107] Qadri S . B., Yang J ., Ratna B. R., Skelton E. F., Hu J . Z. Pressure induced structural transitions in nanometer size particles of PbS. Applied Physics Letters 1996;69(15) 2205-2207.

[108] Jiang J . Z., Olsen J . S ., Gerward L., Frost D., Rubie D., Peyronneau J . Structural stability in nanocrystalline ZnO. Europhysics Letters 2000;50(1)48-53.

[109] Wang H., Liu J. F., He Y., Wang Y., Chen W., Jiang J. Z., Olsen J. S., Gerward L. Highpressure structural behaviour of nanocrystalline Ge. Journal of Physics: Condensed Matter 2007;19(15)156217.

[110] Wang Z., Tait K., Zhao Y., Schiferl D., Zha C., Uchida H., Downs R. T. Size-Induced Reduction of Transition Pressure and Enhancement of Bulk Modulus of AIN Nanocrystals. Journal of Physical Chemistry B 2004;108(31)11506-11508.

[111] Li S., Wen Z., Jiang Q. Pressure-induced phase transition of CdSe and ZnO nanocrystals. Scripta Materialia 2008;59(5) 526-529.

[112] Cui Q., Pan Y., Zhang W., Wang X., Zhang J., Cui T., Xie Y., Liu J., Zou G. Pressureinduced phase transition in GaN nanocrystals. Journal of Physics: Condensed Matter 2002;14(44)11041-11044.

[113] Mujica A., Needs R.J., Munoz A. First-principles pseudopotential study of the phase stability of the III-V semiconductors GaAs and AlAs. Physical Review B 1995;52(12)8881-8892.

[114] Cai J., Chen N. Theoretical study of pressure-induced phase transition in AlAs: From zinc-blende to NiAs structure. Physical Review B 2007;75(17)174116,1-8. 